\title{
EDITORIAL
}

\section{Telling people they are overweight: helpful, harmful or beside the point?}

\section{International Journal of Obesity (2017) 41, 1160-1161; doi:10.1038/ ijo.2017.85}

Weight status misperception refers to when a person's subjective perception of their own or another person's objective weight status is incorrect. Parents of children with overweight and obesity often fail to identify their child as being 'overweight', and in a similar vein, a large number of adults, adolescents and children with overweight or obesity fail to recognise that they are overweight. These observations are not new and have long been presumed to be a concern; if people do not realise they are overweight, how will they change their behaviour to lose weight? For example, the failure of parents to identify their children as overweight has recently been described as 'promoting the silent rise' of obesity, ${ }^{1}$ and new research reported in the International Journal of Obesity suggests that healthcare professionals not notifying children and their families of their 'unhealthy weight status' is a missed opportunity to combat obesity. ${ }^{2}$ These sentiments are echoed in public health intervention approaches. One example is national weight measurement programmes that monitor child weight and notify parents if their child has an 'unhealthy' weight status.

The presumption that ignorance is damaging in this context has face value and is supported by some cross-sectional evidence, as numerous studies have shown that individuals who fail to recognise they are overweight are less likely to be attempting weight loss. However, the best prospective evidence to date suggests that ignorance may be bliss when it comes to overweight and obesity. If ignorance is damaging, then children whose parents fail to identify their overweight status should be the most at risk of further weight gain. The opposite, however, is true: When a parent identifies their child as being overweight, that child is most at risk of future weight gain. ${ }^{3}$ Likewise, if ignorance is damaging, then adolescents and adults with overweight who fail to identify themselves as such should be most at risk of gaining further weight. Again, the opposite is true: it is self-identification of being overweight that is prospectively predictive of greater weight gain. ${ }^{4}$ A recent quasi-experimental study has also shown that, among adolescent females, notification of being overweight was associated with increased weight gain. ${ }^{5}$

What can explain these counter-intuitive findings? Overweight and obesity are widely stigmatised, and knowledge that you possess a characteristic devalued and derogated by society is likely to be psychologically damaging. Theory from social psychology suggests such stigmatisation impairs self-regulation and makes weight management more difficult. ${ }^{6}$ In line with this suggestion, one of the reasons that self-identification of overweight is associated with increased weight gain is because it may place a person at a greater risk of stress-induced overeating. ${ }^{4}$ Likewise, other research has shown that perceived weight discrimination predicts a range of negative health outcomes, including further weight gain. Thus, the stigma attached to identifying as being a person with overweight or obesity may ironically exacerbate these conditions.

Aside from weight gain, evidence links self-identification of overweight to mental health problems. Cross-sectional and longitudinal work examining differences between those who identify vs those who fail to identify themselves as being overweight support this argument and suggest that this process plays out across the lifespan. For example, adolescents who identify as overweight are at higher risk of major depression than those who believe they are of normal weight. ${ }^{7}$ It is plausible that lower psychological well-being predisposes an individual to recognise that they are overweight rather than lower psychological well-being being a consequence of identifying as overweight. Longitudinal data, however, support the former proposition: identifying as being overweight is predictive of poorer psychological outcomes over time. For example, one study found that adolescents who self-identified as overweight (as opposed to not self-identifying) were more likely to experience declines in mental health by young adulthood. ${ }^{8}$ Importantly, the evidence also suggests that whether or not someone identifies as being overweight is predictive of poorer psychological outcomes independently of their objective weight status. See Table 1 for additional studies that link self-identification of overweight to negative health outcomes. These findings suggest that the stigma attached to overweight and obesity not only exacerbates obesity but also damages mental health.

What relevance do these findings have to public health efforts against weight gain and obesity? First, they reinforce the idea that the psychosocial experience of obesity is part of the aetiology of obesity and is likely to be one major contributor to why it is such a devastating condition. Second, they suggest that 'light touch' public health approaches directed at the 'individual', such as informing people that their weight is 'unhealthy', probably have no beneficial effect on health. Moreover, if such approaches do not consider the stigma attached to overweight and obesity they may even be to the detriment of the individual. In terms of successful weight management across the life course, those who identify as overweight fare worse than those who fail to identify as overweight. ${ }^{4}$ A large amount of obesity research to date has focused on intervening at the 'individual' level and few would argue that these efforts have by and large failed; we lack convincing 'individual' level interventions that achieve meaningful weight loss that is maintained in the long term. ${ }^{9}$ This outcome is not surprising when we consider the environment in which the individuals we target reside. Changes to the food environment have been widely accepted as one major cause of the obesity crisis. ${ }^{10}$ With this in mind, if we are serious about tackling obesity we should in part accept that approaches targeted at the individual will be doomed to fail unless the obesogenic food environment is addressed. 'Renormalising' our obesogenic food

Table 1. Additional references

Sonneville et al. ${ }^{11}$

Liechty et al. ${ }^{12}$

Gerards et al. ${ }^{13}$

Essayli et al. ${ }^{14}$

Incollingo Rodriguez et al. ${ }^{15}$

Hunger et al. ${ }^{16}$

Hayward et al. ${ }^{17}$

Atlantis et al. ${ }^{18}$

Vogt Yuan ${ }^{19}$ 
environment will require substantial government investment and policy change. Critically, this process will need to be research led. One benefit of taking aim at the environment, rather than the individual, is that this approach does not stigmatise the individual. This approach also takes aim at the cause and therefore most likely solution to the obesity crisis, our current obesogenic food environment.

\section{CONFLICT OF INTEREST}

The authors declare no conflict of interest.

E Robinson ${ }^{1}$, A Haynes ${ }^{1}$, AR Sutin ${ }^{2}$ and M Daly ${ }^{3,4}$ ${ }^{1}$ Institute of Psychology, Health \& Society, University of Liverpool, Liverpool, UK;

${ }^{2}$ College of Medicine, Florida State University, Tallahassee, FL, USA; ${ }^{3}$ Behavioural Science Centre, Stirling Management School, University of Stirling, Stirling, UK and

${ }^{4}$ UCD Geary Institute, University College, Dublin, Ireland E-mail: eric.robinson@liv.ac.uk

\section{REFERENCES}

1 Hochdorn A, Faleiros VP, Camargo BV, Bousfield AB, Wachelke JF, Quintao IP et al. Obese children are thin in parents' eyes: a psychologically, socially, or culturally driven bias? J Health Psychol 2016; pii: 1359105316676328; e-pub ahead of print 6 November 2016.

2 Hansen AR, Duncan DT, Woo Baidal JA, Hill A, Turner SC, Zhang J. An increasing trend in health-care professionals notifying children of unhealthy weight status: NHANES 1999-2014. Int J Obes 2016; 40: 1480-1485.

3 Robinson E, Sutin AR. Parental perception of weight status and weight gain across childhood. Pediatrics 2016; 137. Available at: http://pediatrics.aappublications.org/ content/early/2016/04/19/peds.2015-3957.

4 Robinson E, Hunger JM, Daly M. Perceived weight status and risk of weight gain across life in US and UK adults. Int J Obes 2015; 39: 1721-1726.

5 Almond D, Lee A, Schwartz AE. Impacts of classifying New York City students as overweight. Proc Natl Acad Sci USA 2016; 113: 3488-3491.

6 Hunger JM, Major B, Blodorn A, Miller CT. Weighed down by stigma: how weightbased social identity threat contributes to weight gain and poor health. Soc Personal Psychol Compass 2015; 9: 255-268.
7 Roberts RE, Duong HT. Perceived weight, not obesity, increases risk for major depression among adolescents. J Psychiatr Res 2013; 47: 1110-1117.

8 Mamun A, Cramb S, McDermott BM, O'callaghan M, Najman JM, Williams GM. Adolescents' perceived weight associated with depression in young adulthood: a longitudinal study. Obesity 2007; 15: 3097-3105.

9 Dombrowski SU, Knittle K, Avenell A, Araujo-Soares V, Sniehotta FF. Long term maintenance of weight loss with non-surgical interventions in obese adults: systematic review and meta-analyses of randomised controlled trials. BMJ 2014; 348: g2646.

10 Swinburn BA, Sacks G, Hall KD, McPherson K, Finegood DT, Moodie ML et al. The global obesity pandemic: shaped by global drivers and local environments. Lancet 2011; 378: 804-814.

11 Sonneville KR, Thurston IB, Milliren CE, Kamody RC, Gooding HC, Richmond TK Helpful or harmful? Prospective association between weight misperception and weight gain among overweight and obese adolescents and young adults. Int $J$ Obes 2016; 40: 328-332

12 Liechty JM, Lee MJ. Body size estimation and other psychosocial risk factors for obesity onset among US adolescents: findings from a longitudinal population level study. Int J Obes 2015; 39: 601-607.

13 Gerards SM, Gubbels JS, Dagnelie PC, Kremers SP, Stafleu A, de Vries NK et al. Parental perception of child's weight status and subsequent BMlz change: the KOALA birth cohort study. BMC Public Health 2014; 14: 291.

14 Essayli JH, Murakami JM, Wilson RE, Latner JD. The impact of weight labels on body image, internalized weight stigma, affect, perceived health, and intended weight loss behaviors in normal-weight and overweight college women. Am J Health Promot 2016. in press.

15 Incollingo Rodriguez AC, Heldreth CM, Tomiyama AJ. Putting on weight stigma: a randomized study of the effects of wearing a fat suit on eating, well-being, and cortisol. Obesity 2016; 24: 1892-1898.

16 Hunger JM, Tomiyama A. Weight labeling and obesity: a longitudinal study of girls aged 10 to 19 years. JAMA Pediatrics 2014; 168: 579-580.

17 Hayward J, Millar L, Petersen S, Swinburn B, Lewis AJ. When ignorance is bliss: weight perception, body mass index and quality of life in adolescents. Int $J$ Obes 2014; 38: 1328-1334 1327.

18 Atlantis E, Ball K. Association between weight perception and psychological distress. Int J Obes 2008; 32: 715-721.

19 Vogt Yuan AS. Body perceptions, weight control behavior, and changes in adolescents' psychological well-being over time: a longitudinal examination of gender. J Youth Adolesc 2010; 39: 927-939. 